\title{
Classification of architectural designs using Deep Learning
}

\section{Devaiah KN, Anita HB}

\begin{abstract}
Architecture style of buildings play's an important role in various aspects. Architectural style or the construction method affects the human health in multiple ways. Many dynasties are ruled India and constructed various types of monuments. So, In this proposed work popular dynasties like Hoysala dynasty, Vijayanagar empire, Mughal empire, Nizam's of Hyderabad, Chalukya dynasty etc. are considered for creating dataset for the work. The architects of those times had really good knowledge about the different scientific methods to be used for construction. This project aims at classification of different architectural styles. Automatic identification of different architectural styles would facilitate different applications. The dataset is manually created by downloading images from various websites. Deep learning, inception v3 master algorithm are used. Experiments are performed using tenser flow and bottle neck files are created for validation. Good recognition rate is achieved with a fewer data set.
\end{abstract}

Keywords: Dynasty, Inception v3, Architecture, Deep Learning, Convolution Neural Network.

\section{INTRODUCTION}

India has great history with respect to the dynasties that ruled this country. Over period of time as the generation changed, the architecture styles of the buildings also changed. In the previous generations the architects followed scientific methods like they built in such a way that the sunlight falls inside through the main door, creation of positive vibes and aura etc. few monuments built with architectural styles that created health benefits. The above mentioned concepts were few benefits of the architectural styles that were followed in the ancient times. Indian sages of the ancient times also had strong scientific knowledge about the architectural style they used to build the various monuments. During those times they used advanced building materials used for the construction. They even used different materials for painting the monuments and for carvings. These painting styles and carvings also help in finding the architectural styles of the monuments. Because they used really good materials for construction even today those monuments are in good condition in spite of different weather and environmental conditions. From those materials will get to know the scientific reason behind the really good condition of the monuments. This is why it is necessary to preserve the information related to these monuments.

Revised Manuscript Received on February 05, 2020

* Correspondence Author

Devaiah K. N., Department of Computer Science, CHRIST (Deemed to be University), Bangalore, India.

Anita H. B., Associate Professor, Department of Computer Science, CHRIST (Deemed to be University), Bangalore, India.

(C) The Authors. Published by Blue Eyes Intelligence Engineering and Sciences Publication (BEIESP). This is an open access article under the CC BY-NC-ND license (http://creativecommons.org/licenses/by-nc-nd/4.0/)
All these information's will give deep insights about the architectural styles of different dynasties. It helps the researchers of different subjects like history, architecture etc, to carryout research in these areas. Image processing is used in different areas to extract features for classification. It finds its application in medical image processing [1], Gabor feature based approach was used to identify different Indian scripts from handwritten document images [2] etc. In this paper, five dynasties that ruled India have been considered. Hoysala dynasty, Vijayanagar empire, Mughal empire, Nizams of Hyderabad, Chalukya dynasty are the once considered in this paper.

Hoysala dynasty: Most of the hindu temple architecture was developed when the Hoysala empires were ruling. The Hoysala architecture follows Dravidian tradition of architecture style. They have their monuments built in different parts of the state. The basic building materials used by Hoysala empires are soapstone.
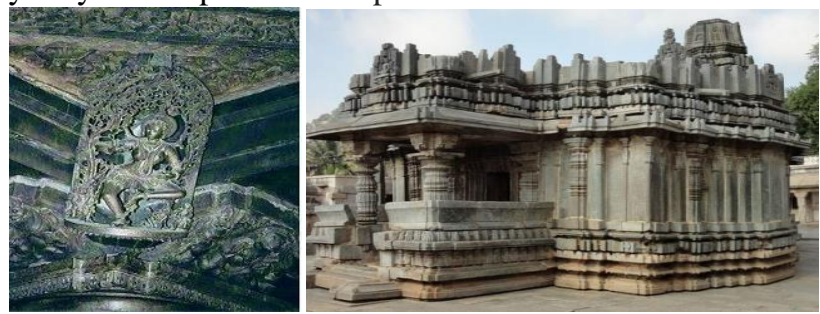

Fig 1.1 Hoysala Dynasty

(a) Madanika at Belur

(b) Akkana Basadi at Shravanbelgola

Vijayanagar empire: The Vijayanagara empire architecture is classified as religious, courtly and civic architecture. The architects also used plaster for giving finishing to the monuments. These materials were used due to their quality, durability, reliability etc. Vijaya vitthala temple, virupaksha temple, lotus mahal, hazara rama temple are few well known temples of Vijayanagara period.
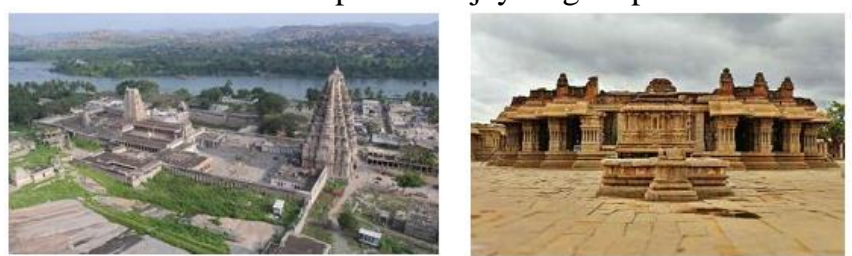

Fig 1.2 Vijayanagara empire

\section{(a) Virupaksha temple \\ (b) Vijaya vitthala temple}

Mughal empire: Mughal architecture reflect to be symmetrical and decorative amalgam of Persian, Turkish, and Indian architecture.

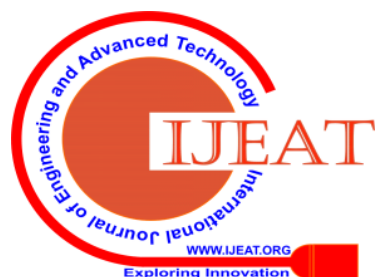


Red sandstone was extensively used as the basic building material. Shah jahan was the one who commissioned the famous Taj mahal which was built in dedication to his wife Mumtaz mahal. It was built using white marble which was also known for its reliability and durability.

Taj mahal, bibi ka maqbara, Kashmiri gate are few famous monuments of Mughal empire.

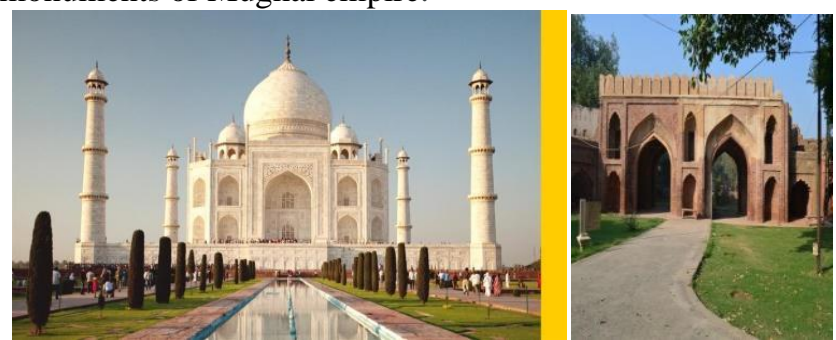

(a) Taj Mahal at Agra

Fig 1.3 Mughal empire

(b)Kashmir Gate

Nizam's of Hyderabad: Nizam's of Hyderabad followed indo-islamic architectural style. Due to this Hyderabad is contemplated to be a heritage city. They had built many mosques and palaces during their ruling period. Most of the historical sites were built during the governance of qutb shahi and asaf jahi. Chowmahalla palace, gulzar houz are famous monuments built by Nizams of Hyderabad.

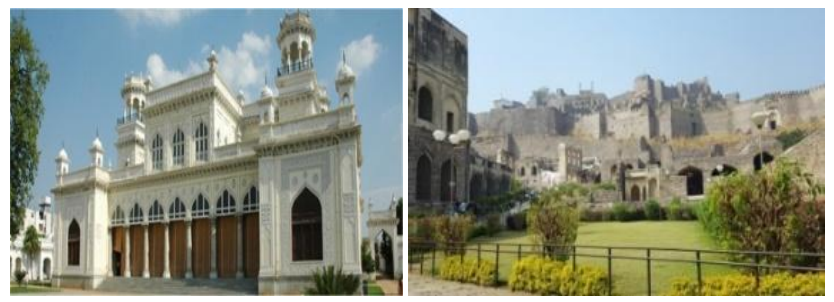

Fig 1.4 Nizam's of Hyderbad

Chalukya dynasty: Chalukya empire period is also known as golden age in the Karnataka history. They were much interested in temple architecture. This dynasty architecture is a blend between Dravidian and Nagara temple architecture. Most of their monuments are rock cut. They have used reddish-golden sandstone as the basic building material. Mallikarjuna temple, sangameshawara temple, ravanphadi cave in Aihole is few well known monuments built during Chalukya period of ruling.

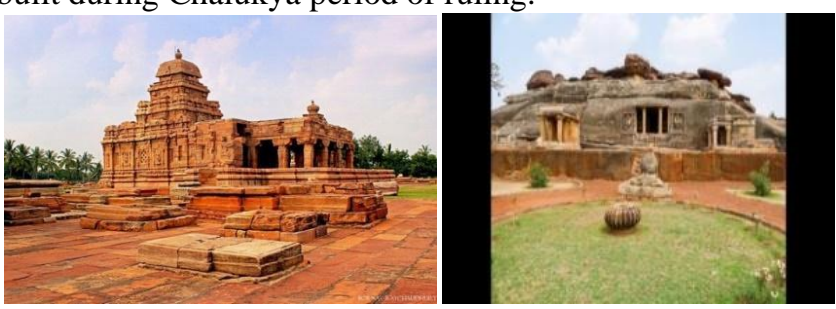

Fig 1.5 Chalukya Dynasty

(a)Mallikarjuna temple

(b)Ravanphadi cave

\section{LITERATURE REVIEW}

In the paper building instance classification using street view images aimed at presenting a framework for building instance classification which will be providing more informative classification maps. This was done for optimum land use classification. [1] In the paper graph convolution neural network for classification of building patterns using spatial vector data highlighted the potential of the GCNN to analyze graph structured spatial data. Additional efforts are to be considered in the future for improving GCNN with respect to deep learning technologies. Efforts should be put in applying GCNN to other analysis task also. [2] In the paper Image Processing of Intelligent Building Environment Based on Pattern Recognition Technology, it aims at studying building contour extraction in satellite images, research and status at home and abroad. Pre-processing technology of images, design and implementation and edge contour technology is also studied. [3] In the paper MultiModel Neural Network for Image Classification a modular architecture for MMNN where there is a dedicated neural network to each class of the problem domain and allowing each of these neural modules to be built according to various paradigm. The modular architecture of MMNN and the execution of benchmarks among a variety of neural network models permits to select the most appropriate paradigm for each thematic class. [4] In the paper classification of radiolucency in dental x-ray image the proposed system is having input as $\mathrm{x}$-ray Images and the outputs are classified into category of radiolucency that it falls under. The classification of the tooth is done by using Multi-Layer Perceptron (MLP), SMO, KNN. Features are extracted using both, Spatial and Frequency domain. Classification is done using Weka tool. [5] In the paper Handwritten script identification from a bi-script document at line level using gabor filters has been proposed for the identification of script from multi script handwritten documents. Gabor filters are used for feature extraction. KNN classifier is used for recognition. They have achieved a recognition rate of 100 percentage. The proposed method is independent of style of hand writing. [6] In this paper the proposed system shows an approach for object and scene retrieval which searches for and localizes all the occurrences of a user outlined object in a video. The object is represented by a set of viewpoint invariant region descriptors so that recognition can proceed successfully despite changes in viewpoint, illumination and partial occlusion. The temporal continuity of the video within a shot is used to track the regions in order to reject unstable regions and reduce the effects of noise in the descriptors. [7] In the paper Visual pattern discovery for architecture image classification and product image search a method has been presented to automatically detect and localize frequent spatial feature configurations, which can successfully describe characteristic features of repetitive objects. Then the graph mining process is used to find patterns under object scaling, rotation, illumination changes. [8] In the paper Image Net Classification with deep convolution neural networks has proposed an approach to classify high resolution images in the imagenet using deep convolutional neural network. [9] In the paper Deep Convolutional Networks for Large Scale Image Recognition is demonstrated. The state-of-the-art performance on the ImageNet challenge dataset can be achieved using a conventional ConvNet architecture with substantially increased depth is mentioned.

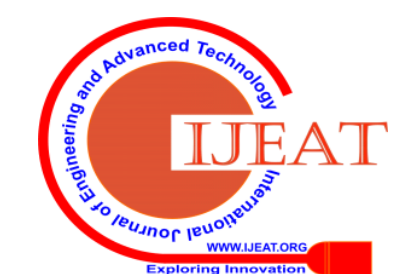


[10] Introduction to Machine Learning by Ethem Alpaydin is used as reference to learn different steps in machine learning like supervised learning, decision tree, dimensionality reduction, clustering, kernel etc.

[11] Data mining concepts and techniques is used as reference to learn about data reduction, classification, visualization etc.

[12] Digital image processing by Rafael c. gonzalez is used as reference material to learn about different topics related to image processing. [13]

\section{DATA COLLECTION}

The dataset is created by collecting images of five different dynasties. The images were downloaded from google from different websites. Images of five different dynasties are collected. Hoysala empire, Vijayanagara empire, Mughal empire, Nizam's of Hyderabad, Chalukya empire are the different classes of images. The constraints used during collecting images are that no presence of letters written, human's standing etc. These constraints were chosen because if there is presence of a human posing in the picture, the extraction of relevant features will be difficult. The presence of water mark on the images might also make a difference if it is considered as a feature. Manually the required portion means architecture image is cropped from the original image. This is done to extract accurate features. Size of each dataset varies for different classes. Size of class Hoysala and Vijayanagara is 173 respectively. The number of images downloaded for Mughal is 104, Chalukya is 310 and Nizam's of Hyderabad is 113. (Table 1).

Table 1:- Dataset

\begin{tabular}{|l|l|}
\hline Dynasty Name & Number of images \\
\hline Hoysala & 173 \\
\hline Vijayanagar & 173 \\
\hline Mughal & 104 \\
\hline Chalukya & 310 \\
\hline Nizam's of Hyderabad & 113 \\
\hline
\end{tabular}

\section{METHODOLOGY}

Inception v3 master model is experimented in this paper. This algorithm is widely used for image recognition and it is also shown to attain greater than $78.1 \%$ accuracy on the ImageNet dataset. The model is a mixture of various ideas developed by multiple researchers over the past years. This is defined to be made as a combination of symmetric and asymmetric building blocks including convolutions, average pooling, max pooling, concats, dropouts, and fully connected layers. Batchnorm is used widely throughout the model and applied to activation inputs. Softmax is used to compute loss. The model must be trained before used to recognize images. This process is done by supervised learning using a large set of labelled images. The inception v3 model can be trained from many different labelled images.

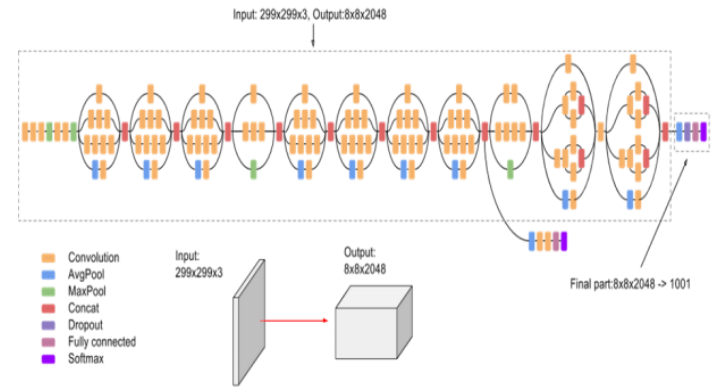

Fig2:- High level diagram of Inception v3 model

\section{FEATURE EXTRACTION}

Convolution nueral network is used for classification of different types of images. In the proposed system inception v3 master is used to build own network and the parameters are modified for maintaining the accuracy. There are hidden layers and from there the features extracted are stored in the bottle neck file. From this the features are given to the softmax layer where the classification is done.

\section{CLASSIFICATION}

This work is based on multi -class classification. Classification of multiple classes with imbalanced dataset is a different challenge than binary classification. Here there are five different classes namely Hoysala, Vijayanagara, Chalukya, Mughal, Nizam's of Hyderabad. The experimental results are obtained for both multi class classification and bi-classification.

\section{EXPERIMENTAL RESULTS}

The multi class classification was applied and obtained an accuracy of 83.4 percentage. Bi-model classification was also carried out for classification. The results are mentioned in Table. 2.

Table 2:- Binary Classification

\begin{tabular}{|c|c|c|}
\hline Class 1 & Class 2 & Accuracy(\%) \\
\hline Chalukya dynasty & Hoysala dynasty & 63.2 \\
\hline Chalukya dynasty & Mughal dynasty & 89.2 \\
\hline Chalukya dynasty & $\begin{array}{c}\text { Nizam of } \\
\text { hyderabad }\end{array}$ & 53.2 \\
\hline Chalukya dynasty & $\begin{array}{c}\text { Vijayanagara } \\
\text { empire }\end{array}$ & 65.3 \\
\hline Hoysala dynasty & Mughal dynasty & 86.5 \\
\hline Hoysala dynasty & $\begin{array}{c}\text { Nizam of } \\
\text { hyderabad }\end{array}$ & 90.1 \\
\hline Hoysala dynasty & $\begin{array}{c}\text { Vijayangara } \\
\text { empire }\end{array}$ & 68.5 \\
\hline Mughal dynasty & $\begin{array}{c}\text { Nizam of } \\
\text { hyderabad }\end{array}$ & 80.8 \\
\hline Mughal dynasty & $\begin{array}{c}\text { Vijayanagara } \\
\text { empire }\end{array}$ & 91.2 \\
\hline $\begin{array}{c}\text { Nizam of } \\
\text { Hyderabad }\end{array}$ & $\begin{array}{c}\text { Vijayanagara } \\
\text { empire }\end{array}$ & 810 \\
\hline
\end{tabular}

As mentioned above, both multi class classification and biclassification is experimented. The experimental results obtained using multi class classification is mentioned below as per the epochs. 
When the model is trained with 30 epochs proposed model obtained accuracy of $58 \%$. Similarly, 93\% accuracy is obtained with 500 epochs. The bi-classification was done and the output test accuracy is tabulated in the above Table 2. From the above table we can infer the accuracy between different classes. The bi classification is done between Chalukya and Hoysala or Chalukya and Vijayanagara empire the accuracy is less. That is because these dynasties have similar style of architecture. When classification is done between Chalukya and Mughal the test accuracy is more because the style architecture between both the dynasties is different. The Chalukya dynasty has Dravidian style of architecture and Mughals have indo Islamic style of architecture due to this good accuracy is obtained. Similarly, Mughal dynasty and Nizam of Hyderabad prediction accuracy is low as both the architectural styles are similar. The model trained with hundred training steps for all the classification.

\section{CONCLUSION}

In this proposed work the classification of architecture using Deep learning is experimented with five different classes and good results are obtained. The average recognition rate of $90 \%$ is obtained. From the result analysis we infer that when the multi class classification is done the accuracy is average. When bi-classification is done it depends on which two classes the classification is done. The classification done between Hoysala dynasty, Vijayanagara empire and Chalukya dynasty the accuracy obtained is very less due to similar architectural styles. When the same classification is done with Mughal dynasty or Nizam of Hyderabad the accuracy is better because of different styles. Inception v3 model is used in this proposed work. Further this system can be updated by adding many other architectural styles of different dynasties. This particular system can also help archaeological department to analyse the various architectures.

\section{REFERENCES}

1. Jian Kang, Marco Körner, Yuanyuan Wang, Hannes Taubenböck, Xiao Xiang Zhu,'Building instance classification using street view images", arxiv, 2018.

2. Xiongfeng Yan, Tinghua Ai, Min Yang, Hongmei Yin," Paper graph convolution neural network for classification of building patterns using spatial vector data", vol 150, ISPRS, Elsevier,2019, p. 259-273.

3. Wei Cai, Xiaodong Wen, Qiu Tu, Xiujuan Guo "Image Processing of Intelligent Building Environment Based on Pattern Recognition Technology", Journal of visual communication and image representation, vol 61, Elsevier, 2019, p. 141-148.

4. Ricardo Jos Machado, Paulo E.C.S.A. Neves "Multi-Mode Neural Network for Image Classification", IEEE, 2002.

5. CJ Britto, HB Anita "Classification of radiolucency in dental xray image", Research journal of pharmacy and technology, volume 12, issue 5,2019, p.2171-2174.

6. GG Rajput, HB Anita "Handwritten script recognition at line level- A multiple features based approach",vol 3 issue 4, IJEIT, 2013,p.90-95.

7. Sivic J, Zisserman A "Video google a text retrieval approach to object matching in videos", IEEE, 2008.

8. Chu W.T, Tsai, M.H "Visual pattern discovery for architecture image classication and product image search", Communications of the ACM, vol 27,2012, p 1-8.

9. Krizhevsky A. Sutskever I, Hinton G "ImageNet classification with deep convolutional networks", Communications of the ACM, vol 6, 2017, p. 6.

10. Simonyan K. Zisserman, A "Very deep convolutional networks for large-scale image recognition", arxiv, vol 6, 2015.
11. Ethem Alpaydin "Introduction to Machine Learning", The MIT Press, 2014.

12. Jaiwei Han "Data mining concepts and techniques", Third Edition, Elsevier ,2012.

13. Rafael C. Gonzalez and Richard E. Woods, Digital image processing, Third Edition Pearson Education, 2008.

14. Saurabh sharma, Priyal Aggarwal " Classification of Indian Monuments into Architectural Styles”, CCIS, Vol 841, 2018

\section{AUTHORS PROFILE}

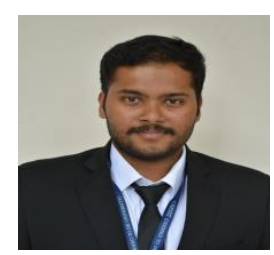

Devaiah K N is currently pursuing his Master's Degree in Computer Science at CHRIST (Deemed to be University), Bengaluru, India. As a part of his current curriculum requirements, he is also a Software Intern working at UTrend Tech Private Ltd. His job profile is front end developer. He completed his under graduation in the year 2018. He has worked on projects like Empower Skilled Laborers, hospital management system etc. For building these projects, React js, html, bootstrap, mysql, mongodb, python and visual basic tools are used. His research interests are mainly focus on the field of Digital Image Processing, Machine Learning and Deep Learnin

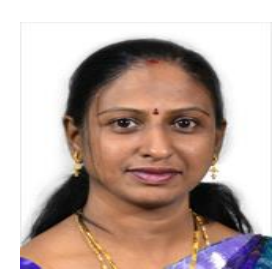

Anita H. B. is presently working as an Associate Professor in Department of Computer Science, CHRIST (Deemed to be University), Bengaluru, India. She has more than 15 years of teaching experience at various institutions. She has received her $\mathrm{PhD}$ degree in 2014 at Gulbarga University, Department of Computer Sciences, Gulbarga, Karnataka, India. Her main research interests are Digital Image Processing, Deep learning, Sound Processing, Machine learning, Medical Image Processing and Signal Processing. Her research contribution is in the classification of images using spatial and frequency domain features. She also supervises research projects. Currently, she is guiding Mphil and PhD students. 